\title{
AVALIAÇÃO NUTRICIONAL E RISCO CARDIOVASCULAR EM EXECUTIVOS SUBMETIDOS A CEOKUP
}

Thelma Fernandes Feltrin Rodrigues*, Sonia Tucunduva Philippi

Trabalho realizado na Faculdade de Saúde Pública da Universidade de São Paulo, São Paulo, SP

\author{
* Correspondência \\ Rua Cristiano Viana, 670 \\ apto. 42 - Pinheiros \\ São Paulo/SP \\ CEP 05411-001 \\ Tel: (11) 3061-3081/ \\ (11) 8124-8409 \\ thenutri@ajato.com.brou \\ thenutri@usp.br
}

\begin{abstract}
RESUMO
Oвjetivo. Avaliar estado nutricional e risco cardiovascular de executivos.

Métodos. Estudo transversal. Foram avaliados 329 executivos de ambos os gêneros, com idade entre 31 e 70 anos, submetidos a check-up de saúde em hospital privado do município de São Paulo. Foram mensuradas as variáveis referentes a peso, estatura e circunferência da cintura (CC) e determinado o Índice de Massa Corporal (IMC). Foram analisados pressão arterial, níveis séricos de colesterol total e frações, triacilglicerol, glicose e ácido úrico. Prática habitual de atividade física foi avaliada por meio do Questionário Internacional de Atividade Física (IPAQ) e o risco cardiovascular pelo Escore de Framingham. A análise de variância e o método de Bonferroni foram utilizados para 0 tratamento estatístico.

Resultados. Média de idade foi 44,6 anos ( $D P=6,8)$, predominando o gênero masculino (89,7\%). Quanto ao estilo de vida, $17 \%$ eram tabagistas e 7,3\% sedentários. Os resultados médios encontrados para os exames clínicos e bioquímicos foram: pressão arterial $117,8 \times 78,6 \mathrm{mmHg}(\mathrm{DP}=12 \times 8,3)$, colesterol total $200,5 \mathrm{mg} / \mathrm{dL}(\mathrm{DP}=35,9)$, LDL-c $121,8 \mathrm{mg} / \mathrm{dL}(\mathrm{DP}=29,9), \mathrm{HDL}-\mathrm{c} 52,2 \mathrm{mg} / \mathrm{dL}(\mathrm{DP}=10,9)$, triacilglicerol $133,7 \mathrm{mg} / \mathrm{dL}(\mathrm{DP}=76,8)$, glicose 96,3 $\mathrm{mg} / \mathrm{dL}(\mathrm{DP}=20,5)$ e ácido úrico 6,0 e 4,2 $\mathrm{mg} / \mathrm{dL}(\mathrm{DP}=1,0$ e 0,9) para homens e mulheres, respectivamente. $O$ IMC médio foi de excesso de peso $\left(26,1 \mathrm{~kg} / \mathrm{m}^{2} ; D P=6,8\right)$. A CC média estava normal entre as mulheres $(79,6 \mathrm{~cm} ; \mathrm{DP}=7,3)$ e indicou risco elevado para doenças crônicas entre os homens $(96,1 \mathrm{~cm} ; \mathrm{DP}=8,9)$. O Risco de Framingham médio foi de $5,7 \%$
\end{abstract}

Conclusão. Os executivos estavam com excesso de peso e apresentaram baixo risco cardiovascular.

UnITERMOS: IMC. Colesterol. Doenças cardiovasculares.

\section{INTRODUÇÃO}

As doenças cardiovasculares constituem um conjunto de afecções com etiologias e manifestações clínicas diversas, de grande importância na estrutura de morbimortalidade dos países desenvolvidos e dos em desenvolvimento, incluindo o Brasil, onde, semelhante às taxas mundiais, as doenças cardiovasculares determinam um terço do total de mortes registradas ${ }^{1,2}$.

A partir do Estudo de Framingham³, elaborou-se o Escore de Framingham, amplamente utilizado e recomendado como método para avaliar o risco que o indivíduo tem em apresentar evento coronariano ${ }^{4}$.

A prática regular de atividade física, bem como a manutenção do peso saudável, vem sendo objeto de estudos que avaliam a influência do estilo de vida na determinação do desenvolvimento de doenças crônico não-transmissíveis (DCNT), demonstrando que esses dois fatores devem ser aplicados nas diferentes estratégias de prevenção de doenças ${ }^{5}$.

Estudos envolvendo indivíduos adultos de diferentes regiões do Brasil avaliaram o risco cardiovascular por meio do Escore de Framingham, indicando que o risco cardiovascular pode ser reduzido por meio do incentivo ao estilo de vida saudávele,7,8.
O presente estudo tem como objetivo avaliar o risco cardiovascular em executivos da Região Sudeste do Brasil, submetidos à avaliação de saúde periódica, e verificar a associação do risco de Framingham segundo gênero, atividade física e estado nutricional.

\section{Métodos}

Trata-se de estudo transversal, utilizando-se amostra de conveniência, em que foram avaliados 329 indivíduos, de ambos os gêneros, com idade entre 31 e 70 anos, submetidos a avaliação de saúde periódica (check-up). Os indivíduos ocupavam cargos de presidência, diretoria, superintendência e gerência de empresas nacionais e multinacionais, não possuiam doença cardiovascular e não faziam uso de medicamento hipolipemiante ou moderador de apetite.

A pesquisa foi aprovada pelos Comitês de Ética em Pesquisa das instituiçõos envolvidas. Todos os indivíduos assinaram o Termo de Consentimento Livre e Esclarecido de acordo com as normas estabelecidas pelo Comitê de Ética em Pesquisa da Universidade de São Paulo (número 065/04).

Para determinação do estado nutricional utilizou-se o Índice de Quetelet ou Índice de Massa Corporal (IMC), dado pela relação: (peso 
$[\mathrm{kg}]) /\left(\right.$ altura $[\mathrm{m}]^{2}$ ) e utilizado os parâmetros da WHO (1995). Os indivíduos foram mensurados por meio de balança digital, tipo plataforma, com estadiômetro, ambos da marca Seca.

Para a atividade física foi utilizado o Questionário Internacional de Atividade Física (IPAQ-08 versão curta), validado para a população brasileira, ${ }^{9}$. Foi auto-preenchido, e respondidas questões relacionadas à freqüência e duração da prática de atividade física de intensidade moderada, vigorosa e de caminhada, realizadas durante uma semana usual.

Para estimativa do risco absoluto de eventos coronarianos foi utilizado o Escore de Framingham, proposto pelo National Colesterol Education Program III (NCEP III). Osfatores de risco que compreendem o Escore de Framingham são: idade, colesterol total, HDL-c, pressão arterial, Diabetes mellitus e tabagismo com uma pontuação espećifica para cada item. O risco é classificado como baixo $(<10 \%)$, médio $(10-20 \%)$ e alto (>20\%).

As amostras sangüíneas para determinação das taxas de glicose, ácido úrico, triacilglicerol, colesterol total e as frações LDL-c e HDL-c foram coletadas após jejum de 12 horas. Os indivíduos foram orientados a fazer uma dieta leve e abster-se do consumo de álcool nas 24 horas antecedentes ao exame. As variáveis lipídicas foram determinadas por método enzimático colorimétrico, automatizado e por meio da fórmula de Friedwald. A glicose e o ácido úrico foram determinados por método enzimático. Os parâmetros usados foram aqueles estabelecidos pelo NCEP III.

Foram calculados, para a análise descritiva dos dados, a média, o desvio padrão, a análise de variância e o método de comparações múltiplas de Bonferroni para verificar a associação entre as variáveis. $O$ nível de significância adotado foi $p<0,05$.

\section{Resultados}

Dos 329 executivos estudados, $89,7 \%$ eram do gênero masculino, sendo a idade média 44,6 anos ( \pm 6,8 anos).

A população de executivos apresentou-se com excesso de peso $\left(\right.$ IMC médio $\left.=26,1 \mathrm{~kg} / \mathrm{m}^{2}\right)$, sendo que mais da metade estava acima do IMC adequado (Tabela I).

Em relação ao tabagismo, $17 \%$ dos executivos referiram fumar. A proporção de fumantes foi maior entre as mulheres $(20,6 \%)$.

De acordo com o IPAQ, 56,4\% dos executivos foram classificados como ativos (mínimo de 20 minutos contínuos de atividade física, três vezes/semana) e 7,3\% sedentários (máximo de dez minutos de atividade física/semana). O restante foi classificado como irregularmente ativo. Os indivíduos ativos apresentaram um IMC menor que os irregularmente ativos e os sedentários $(p=0,006)($ Tabela 2).

As variáveis bioquímicas demonstraram que os executivos estão dentro dos valores considerados adequados para glicose $(96,3 \pm 20,5$ $\mathrm{mg} / \mathrm{dL}$ ), ácido úrico (4,2 \pm 0,9 mg/dL e 6,0 $\pm 1,0 \mathrm{mg} / \mathrm{dL}$ para mulheres e homens, respectivamente), triacilglicerol (133,7 $\pm 76,8 \mathrm{mg} / \mathrm{dL})$, LDL-c $(|2|, 8 \pm 29,9 \mathrm{mg} / \mathrm{dL})$ e HDL-c $(52,2 \pm 10,9 \mathrm{mg} / \mathrm{dL}) .0$ colesterol total se apresentou limítrofe $(200,5 \pm 35,9 \mathrm{mg} / \mathrm{dL})$. A Tabela 3 apresenta a distribuição dos executivos segundo a classificação dos dados bioquímicos.

\begin{tabular}{|c|c|c|c|c|c|c|}
\hline \multirow{2}{*}{$\begin{array}{l}\overline{\text { IMC }} \\
\left(\mathrm{kg} / \mathrm{m}^{2}\right)\end{array}$} & \multicolumn{2}{|c|}{ Mulheres } & \multicolumn{2}{|c|}{ Homens } & \multicolumn{2}{|c|}{ Total } \\
\hline & n & $\%$ & $n$ & $\%$ & $n$ & $\%$ \\
\hline $\begin{array}{l}<25,0 \\
25,0 \mid-30,0 \\
\geq 30,0 \\
\text { Total }\end{array}$ & $\begin{array}{c}28 \\
5 \\
1 \\
34\end{array}$ & $\begin{array}{c}82,4 \\
14,7 \\
2,9 \\
100,0\end{array}$ & $\begin{array}{l}94 \\
171 \\
30 \\
295\end{array}$ & $\begin{array}{l}31,8 \\
58,0 \\
10,2 \\
100,0\end{array}$ & $\begin{array}{l}122 \\
176 \\
31 \\
329\end{array}$ & $\begin{array}{r}37,1 \\
53,5 \\
9,4 \\
100,0\end{array}$ \\
\hline
\end{tabular}

De acordo com o Escore de Framingham, o risco absoluto de eventos coronarianos para os executivos foi considerado baixo $(5,7 \% \pm$ 4,5\%). O valor máximo encontrado foi na ordem de 37\% (Tabela 4).

Utilizando o modelo de análise de variância com dois fatores (gênero e IMC), verificou-se diferença da média do Risco de Framingham entre os gêneros, sendo que os homens apresentaram um risco maior $(6,1 \%)$ do que as mulheres $(I, 7 \%)(p=0,00 I)$, independente da categoria do IMC $(p=0,708)$. Os indivíduos obesos (IMC $\geq$ $30 \mathrm{~kg} / \mathrm{m}^{2}$ ) apresentaram Risco de Framingham superior ao dos eutróficos e com excesso de peso $(p=0,00 \mathrm{I})$.

Os executivos classificados como ativos apresentaram um menor Risco de Framingham (5,4\%) quando comparados com os irregularmente ativos (6,0\%) e os sedentários (6,5\%), porém essa diferença não foi significante na populaçãa estudada $(p=0,269)$.

\section{Discussão}

Os resultados antropométricos indicaram uma maior freqüência de indivíduos com excesso de peso (53,5\%). O peso esteve alterado (IMC $\left.\geq 25 \mathrm{~kg} / \mathrm{m}^{2}\right)$ para a maioria dos executivos $(62,9 \%)$. Comparando com a Pesquisa de Orçamentos Familiares (POF) realizada no Brasil entre os anos de 2002-2003, na qual mais de $40 \%$ dos indivíduos adultos do país apresentam excesso de peso, a proporção de executivos com excesso de peso é maior que a nacional".

Estudo realizado em São Paulo no ano de 2000, envolvendo 214 homens submetidos a check-up, constatou-se que o IMC estava acima de $25 \mathrm{~kg} / \mathrm{m}^{2}$ para $71 \%$ desses indivíduos. O IMC médio encontrado foi de $27,0 \mathrm{~kg} / \mathrm{m}^{2}(\mathrm{DP}=3,7)$, indicando também excesso de peso ${ }^{12}$. Ao analisar o estado nutricional, por meio do IMC, de 65 operários estudados no Rio de Janeiro', verificou-se excesso de peso (Média $=25,0 \mathrm{~kg} / \mathrm{m}^{2} ; \mathrm{DP}=4,66$ ), sendo que quase metade dos indivíduos tiveram IMC acima de $25,0 \mathrm{~kg} / \mathrm{m}^{2}$.

Estudando mais de mil pessoas em uma cidade da região sudeste do Brasil, observou-se que 26, I\% estavam com o IMC maior que $27,0 \mathrm{~kg} / \mathrm{m}^{2}{ }^{14}$. Matos e Ladeia, em estudo conduzido no estado da Bahia, envolvendo I 26 indivíduos de uma comunidade rural, indicaram uma prevalência de obesidade na ordem de 7,9\% e $27,8 \%$ para excesso de peso 6 .

Esses estudos vêm corroborar com a preocupação mundial em se estabelecer programas que incentivem mudanças no estilo de vida, visando maior controle de peso, uma vez que a obesidade é um dos determinantes para o desenvolvimento de doenças crônicas não transmissíveis ${ }^{5}$.

Apesar do IMC não levar em consideração a composição corporal do indivíduo e dessa forma, não revelar o excesso de gordura corporal, 
RODRIGUES TFF ET AL.

\begin{tabular}{|c|c|c|c|c|c|c|}
\hline \multirow[b]{2}{*}{ Idade média em anos (DP) } & \multicolumn{2}{|c|}{ Mulheres } & \multicolumn{2}{|c|}{ Homens } & \multicolumn{2}{|c|}{ Total } \\
\hline & $\mathrm{n}$ & Média(DP) & $n$ & Média(DP) & $n$ & Média (DP) \\
\hline & 34 & $42,6(5,3)$ & 295 & $44,8(7,0)$ & 329 & $44,6(6,8)$ \\
\hline & $\mathrm{n}$ & $\%$ & $\mathrm{n}$ & $\%$ & $\mathrm{n}$ & $\%$ \\
\hline $\begin{array}{l}\text { Tabagismo } \\
\text { Tabagistas } \\
\text { Não-tabagistas } \\
\text { Total }\end{array}$ & $\begin{array}{c}7 \\
27 \\
34\end{array}$ & $\begin{array}{l}20,6 \\
79,4 \\
100,0\end{array}$ & $\begin{array}{l}49 \\
246 \\
295\end{array}$ & $\begin{array}{l}16,6 \\
83,4 \\
100,0\end{array}$ & $\begin{array}{l}56 \\
273 \\
329\end{array}$ & $\begin{array}{l}17,0 \\
83,0 \\
100,0\end{array}$ \\
\hline $\begin{array}{l}\text { Prática de atividade física } \\
\text { Ativo } \\
\text { Irregulamenteativo } \\
\text { Sedentário } \\
\text { Total }\end{array}$ & $\begin{array}{l}18 \\
14 \\
2 \\
34\end{array}$ & $\begin{array}{l}52,9 \\
41,2 \\
5,9 \\
100,0\end{array}$ & $\begin{array}{l}167 \\
105 \\
22 \\
294\end{array}$ & $\begin{array}{l}56,8 \\
35,7 \\
7,5 \\
100,0\end{array}$ & $\begin{array}{l}185 \\
119 \\
24 \\
328\end{array}$ & $\begin{array}{l}56,4 \\
36,3 \\
7,3 \\
100,0\end{array}$ \\
\hline
\end{tabular}

é um método muito utilizado como indicador do estado nutricional em pesquisas epidemiológicas, devido o seu baixo custo, facilidade de mensuração e relação com morbimortalidade ${ }^{15}$.

O número de sedentários na população estudada ficou abaixo do encontrado por Matsudo et al. (2002) que, avaliando o nível de atividade física, por meio do IPAQ, em 200 I indivíduos de 29 cidades do Estado de São Paulo, com idade entre 14 e 77 anos, encontraram $55,3 \%$ de indivíduos sedentários pertencentes à classe social A. De acordo com os critérios propostos no IPAQ, sedentário corresponde à prática de no máximo dez minutos contínuos de atividade física durante a semana ${ }^{10}$.

Dos executivos no presente estudo, 56,4\% classificaram-se como ativos. A classificação ativa corresponde à prática de exercícios físicos por, no mínimo, 20 minutos contínuos, três vezes por semana, de acordo com as atividades relacionadas no IPAQ.

Utilizando outro método para avaliar a prática de atividade física verificou-se freqüência semelhante. Em uma amostra de 126 adultos, $56,5 \%$ foi considerada ativa, sendo que a classificação ativa englobou as pessoas que praticavam atividade física no mínimo três vezes por semana ou tinham atividade ocupacional que dispendia altas taxas de calorias (carpinteiro, agricultor, carvoeiro) ${ }^{6}$.

Em estudo retrospectivo, avaliando a presença de fatores de risco em 2546 asiáticos, verificou-se que a prevalência de inatividade física era maior entre os casos de morte quando comparados com os indivíduos vivos. O estudo ainda revelou que os homens mostraramse mais ativos em relação às mulheres ${ }^{16}$.

Sendo a atividade física um importante componente do estilo de vida das pessoas e contribuindo na prevenção das doenças cardiovasculares, o sedentarismo não se apresentou como um fator de risco para a maioria dos executivos estudados.

Ao analisar a diferença do IMC entre as categorias de atividade física, verificou-se que à medida que houve aumento da freqüência e da duração da atividade física, menor foi o IMC desses indivíduos, reforçando assim o importante papel da prática de atividade física para diminuição do balanço energético e conseqüente redução ou manutenção do peso ${ }^{17}$.

\begin{tabular}{|c|c|c|c|c|c|c|}
\hline \multicolumn{7}{|c|}{$\begin{array}{c}\text { Tabela 3 - Distribuição dos indivíduos, segundo } \\
\text { gênero e risco de Framingham (RF) }\end{array}$} \\
\hline R.F. & & eres & & iens & & \\
\hline$\left(\mathrm{kg} / \mathrm{m}^{2}\right)$ & $n$ & $\%$ & $n$ & $\%$ & $n$ & $\%$ \\
\hline $\begin{array}{l}<10 \text { (baixo) } \\
10 \mid-20 \text { (médio) } \\
\geq 20 \text { (alto) } \\
\text { Total }\end{array}$ & $\begin{array}{c}34 \\
0 \\
0 \\
34\end{array}$ & $\begin{array}{c}100,0 \\
0,0 \\
0,0 \\
100,0\end{array}$ & $\begin{array}{c}248 \\
39 \\
8 \\
295\end{array}$ & $\begin{array}{c}84,1 \\
13,2 \\
2,7 \\
100,0\end{array}$ & $\begin{array}{c}282 \\
39 \\
8 \\
329\end{array}$ & $\begin{array}{c}85,7 \\
11,9 \\
2,4 \\
100,0\end{array}$ \\
\hline
\end{tabular}

O Seven Countries Study investigou a associação entre atividade física e a gordura corporal, utilizando para isso a medição da dobra cutânea subescapular. Os resultados apontaram relação inversa entre a prática de atividade física e os depósitos de gordura corporal. Resultado similar, mas com menor poder de associação foi observado com o $\mathrm{IMC}^{18}$.

Os valores para colesterol total (Média $=200,5 \mathrm{mg} / \mathrm{dL} ; \mathrm{DP}=35,9$ ) assemelham-se ao de outros estudos. Durante check-up anual dos funcionários de uma indústria japonesa encontrou-se colesterol total médio de $193 \mathrm{mg} / \mathrm{dL}(\mathrm{DP}=34)^{19}$. Estudo realizado com 672 indivíduos, com idade média de 47 anos, do município de São José do Rio Preto(SP), encontrou valor médio para o colesterol total de $192,5 \mathrm{mg} / \mathrm{dL}(\mathrm{DP}=48,9)$. Sabe-se que o colesterol guarda relação direta com as doenças cardiovasculares, observando-se riscos cada vez mais elevados quanto maior a colesterolemia, principalmente acima de $200 \mathrm{mg} / \mathrm{dL}$. No entanto, a média encontrada está na faixa inferior da classificação limítrofe, amenizando o risco quando isolado de outros fatores ${ }^{20}$.

No que se refere ao valor médio para triacilglicerol ( $133,7 \mathrm{mg} / \mathrm{dL}$, $\mathrm{DP}=76,8)$, corresponde aos resultados de Matos e Ladeia ${ }^{6}$ que avaliaram 126 indivíduos de uma comunidade rural do Estado da Bahia, Brasil, e observaram comportamento semelhante em relação a esse lipídio (Média $=128,6 \mathrm{mg} / \mathrm{dL}, \mathrm{DP}=76,5$ ). A proporção de diabéticos também foi similar (4\%) aos 2,7\% de indivíduos com esse diagnóstico no presente estudo. No entanto, 19,2\% dos executivos apresentaram pré-diabetes, necessitando de intervenção dietética. 


\begin{tabular}{|c|c|c|c|c|c|c|}
\hline \multirow{2}{*}{$\begin{array}{l}\text { Níveis Séricos } \\
(\mathrm{mg} / \mathrm{dl})\end{array}$} & \multicolumn{2}{|c|}{ Mulheres } & \multicolumn{2}{|c|}{ Homens } & \multicolumn{2}{|c|}{ Total } \\
\hline & $n$ & $\%$ & $n$ & $\%$ & $n$ & $\%$ \\
\hline $\begin{array}{l}\text { Glicose } \\
<100 \\
100 \mid-1260 \\
\geq 126 \\
\text { Total }\end{array}$ & $\begin{array}{c}34 \\
0,0 \\
0 \\
34\end{array}$ & $\begin{array}{c}100,0 \\
63 \\
0,0 \\
100,0\end{array}$ & $\begin{array}{c}223 \\
21,4 \\
9 \\
295\end{array}$ & $\begin{array}{c}75,5 \\
63 \\
3,1 \\
100,0\end{array}$ & $\begin{array}{c}257 \\
19,2 \\
9 \\
329\end{array}$ & $\begin{array}{c}78,1 \\
2,7 \\
100,0\end{array}$ \\
\hline \begin{tabular}{l}
\multicolumn{2}{l}{ Ácido úrico } \\
\begin{tabular}{l|l}
$M^{*}$ & $H^{*}$ \\
$\leq 5,2$ & $\leq 7,0$ \\
$>5,2$ & $>7,0$ \\
Total
\end{tabular} \mid
\end{tabular} & $\begin{array}{l}28 \\
6 \\
34\end{array}$ & $\begin{array}{l}82,4 \\
17,6 \\
100,0\end{array}$ & $\begin{array}{c}255 \\
40 \\
295\end{array}$ & $\begin{array}{l}86,4 \\
13,6 \\
100,0\end{array}$ & $\begin{array}{c}283 \\
46 \\
329\end{array}$ & $\begin{array}{l}86,0 \\
14,0 \\
100,0\end{array}$ \\
\hline $\begin{array}{l}\text { Triacilglicerol } \\
<\mid 50 \\
\geq \quad \mid 50 \\
\text { Total }\end{array}$ & $\begin{array}{c}31 \\
3 \\
34\end{array}$ & $\begin{array}{c}91,2 \\
8,8 \\
100,0\end{array}$ & $\begin{array}{l}198 \\
97 \\
295\end{array}$ & $\begin{array}{l}67,1 \\
32,9 \\
100,0\end{array}$ & $\begin{array}{l}229 \\
100 \\
329\end{array}$ & $\begin{array}{l}69,6 \\
30,4 \\
100,0\end{array}$ \\
\hline $\begin{array}{l}\text { Colesterol total } \\
<200 \\
200 \mid-240 \\
\geq 240 \\
\text { Total }\end{array}$ & $\begin{array}{c}18 \\
13 \\
3 \\
34\end{array}$ & $\begin{array}{c}53,0 \\
38,2 \\
8,8 \\
100,0\end{array}$ & $\begin{array}{l}153 \\
105 \\
37 \\
295\end{array}$ & $\begin{array}{l}51,9 \\
35,6 \\
12,5 \\
100,0\end{array}$ & $\begin{array}{l}171 \\
118 \\
40 \\
329\end{array}$ & $\begin{array}{l}52,0 \\
35,9 \\
12,1 \\
100,0\end{array}$ \\
\hline $\begin{array}{l}\text { LDL-C } \\
<130 \\
130 \mid-160 \\
\geq 160 \\
\text { Total }\end{array}$ & $\begin{array}{c}23 \\
8 \\
3 \\
34\end{array}$ & $\begin{array}{c}67,7 \\
23,5 \\
8,8 \\
100,0\end{array}$ & $\begin{array}{l}188 \\
76 \\
28 \\
292\end{array}$ & $\begin{array}{c}64,4 \\
26,0 \\
9,6 \\
100,0\end{array}$ & $\begin{array}{l}211 \\
84 \\
31 \\
326\end{array}$ & $\begin{array}{c}64,7 \\
25,8 \\
9,5 \\
100,0\end{array}$ \\
\hline $\begin{array}{l}\text { HDL-C } \\
<40 \\
40|-| 60 \\
>\quad 60 \\
\text { Total }\end{array}$ & $\begin{array}{l}1 \\
13 \\
20 \\
34\end{array}$ & $\begin{array}{c}3,0 \\
38,2 \\
58,8 \\
100,0\end{array}$ & $\begin{array}{c}36 \\
218 \\
41 \\
295\end{array}$ & $\begin{array}{l}12,2 \\
73,9 \\
13,9 \\
100,0\end{array}$ & $\begin{array}{c}37 \\
231 \\
61 \\
329\end{array}$ & $\begin{array}{l}11,3 \\
70,2 \\
18,5 \\
100,0\end{array}$ \\
\hline
\end{tabular}

O valor médio da glicose de jejum $(96,3 \mathrm{mg} / \mathrm{dL}, \mathrm{DP}=20,5)$ apresentou-se próximo ao limite superior de normalidade ( $99 \mathrm{mg} / \mathrm{dL}$ ), ultrapassando este quando analisado juntamente com o desvio padrão. Esse achado coincide com o estudo de Kurata ${ }^{19}$ que encontrou valor médio de $98 \mathrm{mg} / \mathrm{dL}(\mathrm{DP}=18 \mathrm{mg} / \mathrm{dL})$ entre 6444 homens durante check-upanual.

Dentre os fatores de risco identificados que contribuem para o desenvolvimento das doenças cardiovasculares, a hiperuricemia também pode ser um deles. $\mathrm{O}$ ácido úrico sérico em níveis elevados pode aumentar a agregação plaquetária e intensificar a tendência trombótica ${ }^{2 !}$.

Nos dados do Framingham Heart Study verificou-se relação entre o ácido úrico sérico e o risco coronário. Os níveis desse elemento relacionaram-se significantemente com a incidência de doenças coronarianas, podendo também estar relacionado com a pressão arterial ${ }^{21}$.

No presente estudo os homens apresentaram um valor médio do ácido úrico superior às mulheres $(6,0 \mathrm{mg} / \mathrm{dL}$ e 4,2 mg/dL, respectiva- mente), semelhante ao encontrado no Framingham Heart Study. Os parâmetros de normalidade diferem entre os gêneros. Nesse caso, os valores médios estavam dentro do valor desejável para mulheres e homens. A hiperuricemia esteve presente em 14\% dos executivos.

No Brasil, poucos foram os estudos que utilizaram o Escore de Framingham para a determinação do risco cardiovascular. Os executivos estudados apresentaram baixo risco, segundo o RF (Média =5,7\%; $\mathrm{DP}=4,5)$ semelhante a estudo envolvendo 100 indivíduos que viviam no estado do Amazonas. Comparou-se o perfil lipídico e o risco cardiovascular das pessoas que viviam na região urbana e ribeirinha e em ambas as populações houve baixo risco cardiovascular, ou seja, um RF inferior a 10\%?.

Estudo envolvendo 1.712 indivíduos residentes na cidade de Bambuí, Estado de Minas Gerais, também encontrou baixo risco cardiovascular, segundo o RF. Da população com idade entre 30 e 59 anos, mais da metade não ultrapassou a pontuação de $5 \%$, ressaltando que à medida que a população envelhece, existe a tendência dessa porcentagem aumentar. Verificou-se que mais de $25 \%$ das pessoas 
RODRIGUES TFF ET AL.

com idade entre 60 e 74 anos tiveram um risco cardiovascular maior que $30 \%{ }^{8}$.

Matos e Ladeia ${ }^{6}$ avaliaram os fatores de risco cardiovascular por meio do Escore de Framingham, e em 126 adultos a prevalência foi de $39,7 \%$ de indivíduos com alto risco.

A evolução da mortalidade no Brasil, segundo o Ministério da Saúde ${ }^{22}$, apontou que nas últimas décadas a principal causa de morte foi decorrente de doenças do aparelho circulatório, para ambos os gêneros, em todas as regiões brasileiras.

Todas as mulheres envolvidas no presente estudo apresentaram baixo risco cardiovascular, segundo o RF. Os homens tiveram um risco significantemente maior quando comparadas às médias entre os gêneros, Barreto et al. ${ }^{8}$, aplicando o Escore de Framingham em 547 adultos de ambos os gêneros, residentes em um município de Minas Gerais, encontraram maior prevalência de todos os fatores de risco cardiovascular entre os homens.

Castanho et al. ${ }^{23}$, estudando 873 indivíduos adultos, de ambos os sexos e de diferentes classes socioeconômicas de um município de São Paulo, verificaram que existem diferenças significativas entre os gêneros, em relação aos fatores de risco para doenças cardiovasculares. As mulheres apresentaram maior risco, uma vez que foi identificado entre elas, maior número de casos de hipertensão, diabetes, hipercolesterolemia e sedentarismo.

Para o cálculo do RF, o peso corporal não é considerado, no entanto é consenso que a flutuação do peso está associada com a saúde do indivíduo. No presente estudo observou-se que o RF foi significantemente maior entre os indivíduos com excesso de peso e obesidade, aumentando ainda mais o risco dessas pessoas em desenvolver doenças cardiovasculares.

Analisando a evolução do peso ao longo de 32 anos de acompanhamento de mais de cinco mil homens e mulheres residentes em Framingham, estudiosos observaram que o aumento do peso tem um papel influente no desenvolvimento de doenças crônicas, dentre elas as cardiovasculares ${ }^{24}$.

Ao examinar o impacto da gordura abdominal e do IMC na morbimortalidade em uma coorte do Estudo de Framingham, envolvendo 5.209 indivíduos acompanhados por 24 anos, os resultados apontaram associação entre ambas as medidas e o aumento das taxas de desenvolvimento de doenças cardiovasculares ou aumento do risco de ocorrer tais eventos ${ }^{25}$. Dessa forma, confirma-se a relação do ganho de peso como fator de risco cardiovascular.

Apesar de a diferença encontrada do risco cardiovascular não ser significativa entre os indivíduos ativos e os sedentários, a atividade física é um indicador que pode melhorar o perfil de risco cardiovascular. Matos e Ladeia ${ }^{6}$ observaram em seu estudo que no grupo com maior freqüência de atividade física houve uma tendência inversa de obesidade abdominal e melhores níveis de lipídios séricos.

Em estudo utilizando diferente metodologia para determinar freqüência e intensidade de atividade física, demonstrou-se haver associação desta com o perfil lipídico. Avaliando o nível de atividade física de lazer de 537 homens saudáveis, por meio de questionário contendo 64 sugestões de atividades, encontrou-se forte associação entre indivíduos que praticaram exercícios de maior intensidade e melhor perfil lipídico, juntamente com menor índice aterogênico ${ }^{26}$.

\section{Conclusão}

Os executivos apresentaram baixo risco cardiovascular; sendo que os homens tiveram maior risco do que as mulheres. Quanto maior o IMC, maior foi o Risco de Framingham e quanto maior a prática de atividade física, menor o risco. As mulheres apresentaram menor risco para doenças cardiovasculares, uma vez que tiveram o IMC e a CC dentro da normalidade Os executivos mostraram-se ativos e não-tabagistas, com diagnóstico de excesso de peso, segundo o IMC. Os exames bioquímicos apresentaram-se normais, assim como a pressão arterial. Recomenda-se, no entanto, monitoramento constante deste grupo de executivos com orientações específicas sobre saúde, qualidade de vida e nutrição.

\section{Conflito de interesse: não há}

\section{SUMMARY}

Nutritional evaluation and cardiovascular Risk in EXECUTIVES SUBMITTED TO A CHECK-UP

OBIECTIVE. To evaluate the nutritional status and cardiovascular risk among executives.

METHODS. In a cross-sectional study. 329 executives of both genders, aged 3 I to70 years, were evaluated during a health check-up in a private hospital in the city of São Paulo. The variables related to weight, heightand waist circumference were measured and Body Mass Index-BMI $\left(\mathrm{kg} / \mathrm{m}^{2}\right)$ was determined. Blood pressure and serum levels of total cholesteroland fractions, triacylglycerol, glucose and uric acid were analyzed. Physical activity was evaluated by the International Physical Activity Questionnaire (IPAQ) and cardiovascular risk was estimated by the Framingham Scoring. The variance analysis and the Bonferronimethods were used for statistical treatment.

RESULTS. The mean age was $44.6(S D=6.8)$, with the male gender predominating (89.7\%). As for lifestyle, $17 \%$ were tobacco smokers and $7.3 \%$ sedentary. The mean values found in the clinical and biochemical exams were the following: blood pressure of $117 \times 78.6 \mathrm{mmHg}$ $(S D=12 \times 8.3)$, total cholesterol of $200.5 \mathrm{mg} / \mathrm{dL}(S D=35.9), L D L$-cof 121.8 $\mathrm{mg} / \mathrm{dL}(S D=29.9), H D L-c$ of $52.2 \mathrm{mg} / \mathrm{dL}(S D=10.9)$, triacylglycerol of $133.7 \mathrm{mg} / \mathrm{dL}(S D=76.8)$, glucose of $96.3 \mathrm{mg} / \mathrm{dL}(S D=20.5)$ and uric acid 4.2 and $6.0 \mathrm{mg} / \mathrm{dL}(S D=1.0$ and 0.9$)$ formen and women respectively. The BMI mean for all executives was classified as overweight $\left(26.1 \mathrm{~kg} / \mathrm{m}^{2}\right.$; $S D=6.8)$. Waist circumference was normal in women $(79.6 \mathrm{~cm} ; S D=7.3)$ and indicated a high risk level of chronic disease for the men $(96.1 \mathrm{~cm}$; $S D=8.9)$. The average Framingham Risk was 5.7\%.

CONCLUSION. The executives were overweight and presented low cardiovascular risk, according to the Framingham Scoring. [Rev Assoc Med Bras 2008; 54(4): 322-7]

KEY WORDS: BMI. Cholesterol. Cardiovascular diseases.

\section{REFERÊNCIAS}

I. Lotufo PA, Lolio CA. Tendências de evolução da mortalidade por doenças cardiovasculares: o caso do estado de São Paulo. In: Monteiro CA, organizador. Velhos e novos males da saúde no Brasil. São Paulo: Hucitec; 1995. p.279-88. 
2. World Health Organization. Diet, nutrition and the prevention of chronic diseases. Report. Geneva: World Health Organization; 2003. (Technical Report Series 9|6).

3. D'Agostino RB, Grundy S, Sullivan LM, Wilson P. Validation of the Framingham heart disease prediction scores: results of a multiple ethnic groups investigation. JAMA. 200 I;286(2): I 80-7.

4. NCEP: Executive Summary of the Third Report of the National Cholesterol Education Program. Expert panel on detection, evaluation, and treatment of high blood cholesterol in adults (Adult Treatment Panel III). JAMA. 200I;285:2486-97.

5. World Health Organization. Global strategy on diet, physical activity and health. Geneva: World Health Organization; 2004. (World Health Assembly 57).

6. Matos AC, Ladeia AM. Assessment of cardiovascular risk factors in a rural community in the Brazilian state of Bahia. Arq Bras Cardiol. 2003;8I (3):297-302

7. Feio CMA, Fonseca FAH, Rego SS, Feio MNB, Elias MC, Costa EAS, et al. Perfil lipídico e risco cardiovascular em amazônidas. Arq Bras Cardiol. 2003;8I(6):592-5.

8. Barreto SM, Passos VMA, Cardoso ARA, Lima-Costa MF. Quantifying the risk of coronary artery disease in a community. The Bambuí Project. Arq Bras Cardiol. 2003;8I(6). Disponível em: http://www.scielo.br/scielo. [citado 2004 jun 24].

9. Matsudo S, Araújo T, Matsudo V, Andrade D, Andrade E, Oliveira LC, et al. Questionário internacional de atividade física (IPAQ): estudo de validade e reprodutibilidade no Brasil. Rev Bras Ativ Fis Saúde $2001 ; 6(2): 5-18$

10. Matsudo SM, Matsudo VR, Araújo T, Andrade D, Andrade E, Oliveira L et al. Nível de atividade física da população do Estado de São Paulo: análise de acordo com o gênero, idade, nível socioeconômico, distribuição geográfica e de conhecimento. Rev Bras Ciênc Mov. 2002; I 0(4):41-50.

II. IBGE. Instituto Brasileiro de Geografia e Estatística. Pesquisa de Orçamentos Familiares 2002-2003. Análise da disponibilidade domiciliar de alimentos e do estado nutricional no Brasil. Rio de Janeiro, 2004. Disponível em: http://www.ibge.gov.br. [citado 2005 maio 5].

12. Rodrigues TFF, Vasconcelos MIL, Araújo KNEH, Larsson EJ, Miyahira NS Avaliação do hábito alimentar de executivos atendidos no check-up do Hospital Alemão Oswaldo Cruz. In: Anais do Simpósio Sul-Brasileiro de Alimentação e Nutrição; 2000. Florianópolis; 2000.

13. Castro MTB, Anjos LA, Lourenço PM. Padrão dietético e estado nutricional de operários de uma empresa metalúrgica do Rio de Janeiro, Brasil. Cad Saude Publica. 2004;20(4):926-34.

14. Fornés NAS, Martins IS, Meléndez GV, Latorre MRDO. Escores de consumo alimentar e níveis lipêmicos em população de São Paulo, Brasil. Rev Saude Publica. 2002;36( I): I 2-8.
15. Anjos LA. Indice de massa corporal (massa corporal.estatura-2) como indicador do estado nutricional de adultos: revisão da literatura. Rev Saude Publica. 1992;26(6):431-6.

16. Collins VR, Dowse GK, Cabealawa S, Ram P, Zimmet PZ. High mortality from cardiovascular disease and analysis of risk factors in Indian and Melanesian Fijians. Int J Epidemiol. 1996;25(I):59-69.

17. Mendonça CP, Anjos LA. Aspectos das práticas alimentares e da atividade física como determinantes do crescimento do sobrepeso/obesidade no Brasil. Cad Saude Publica 2004;20(3):698-709.

18. Kromhout D, Bloemberg B, Seidell JC, Nissinen A, Menotti A. Physical activity and dietary fiber determine population body fat levels: the Seven Countries Study. Int J Obes Relat Metab Disord. $2001 ; 25(3): 301-6$.

19. Kurata C. Strategy for primary prevention of coronary heart disease based on results of health check-up. Sangyo Eiseigaku Zasshi. 2000;42(3):81-7.

20. Nicolau JC, Bechara DL, Nascimento SDG, Greco OT, Jacob JLB, Lorga AM. Perfil do colesterol na cidade de São José do Rio Preto. Arq Bras Cardiol. 1992;59(6):433-40

21. Brand FN, McGee DL, Kannel WB, Stokes J, Castelli WP. Hyperuricemia as a risk factor of coronary heart disease: The Framingham Sutdy. Am J Epidemiol. 1985; | 2 I ( I): | | -8.

22. Ministério da Saúde. Secretaria de Vigilância em Saúde. Saúde Brasil 2004: uma análise da situação de saúde. Disponível em: http:// dtr200 I.saude.gov.br/svs/pub/ pub48.htm. [citado 2005 jan I 5].

23. Castanho VS, Oliveira LS, Pinheiro HP, Oliveira HCF, Faria EC. Sex differences in risk factors for coronary heart disease: a study in a Brazilian population. BMC Public Health. 200I;I(3). Disponível em: http:// www.biomedcentral.com/. [citado 2005 jan 15].

24. Lissner L, Odell PM, D 'Agostinho RB, Stokes J, Kreger BE, Belanger AJ, et al. Variability of body and health outcomes in the Framingham population. N Engl J Med. 1991;324(26): I 839-44.

25. Kannel WB, Cupples A, Ramaswami R, Sokes J, Kreger BE, Higgins M. Regional obesity and risk of cardiovascular disease: The Framingham Study. J Clin Epidemiol. 1991;44(2): I 83-90.

26. Marrugat J, Elosua R, Covas MI, Molina L, Rubiés-Prat J. Amount and intensity of physical activity, physical fitness, and serum lipids in men. Am J Epidemiol. 1996; |43(6):562-7.

Artigo recebido: 29/05/07

Aceito para publicação: 25/03/08 